${ }^{1}$ Federal University of Rio de Janeiro, School of Dentistry, Department of Dental Clinic, Rio de Janeiro, Rio de Janeiro, Brazil.

${ }^{2}$ Federal University of Rio de Janeiro, Technology Center, Department of Nuclear Instrumentation Laboratory, Rio de Janeiro, Rio de Janeiro, Brazil.
Corresponding author: Heloisa Gusman

Rua Prof. Rodolpho Paulo Rocco $325 / 2^{\circ}$ andar, llha da Cidade Universitária, Rio de Janeiro. Zip Code: 21941-913. Brazil.

E-mail: heloisa.gusman@odonto.ufrj.br Phone: 5521996312426

Received: July 9, 2020

Accepted: January 21, 2021

\section{Retreatment of mesial roots of mandibular molars filled with resin-based and bioceramic sealers}

\author{
Marina de Almeida Salim ${ }^{1}$, Fabiola Ormiga ${ }^{1}$ (D), Ricardo \\ Tadeu Lopes ${ }^{2}$ (D), Heloisa Gusman ${ }^{1, *}$ (D)
}

Aim: The aim of this study was to evaluate, by micro-computed tomography (micro-CT) analysis, the remaining filling material during endodontic retreatment performed with Protaper retreatment without solvent. Methods: Forty mandibular molars were divided into two groups ( $n=20)$ according to the sealer used in the obturation: the bioceramic TotalFill BC (TF) or the resin-based AH Plus (AHP). The specimens were scanned before instrumentation, after obturation and after filling removal. Only the mesial roots were analysed. The filling volumes and the remaining filling material were calculated in the entire root canal and in the cervical, middle and apical thirds. Results: The volume of obturation and the volume of remaining filling material in the entire root canal and in the cervical, middle and apical thirds of the canal between the groups were not statistically different (independent $t$-test, $p>0.05)$. In the AHP group, there was a higher percentage of remaining filling material in the middle third than in the cervical third $(p<0.05)$. Conclusion: The filling material could not be entirely removed from any specimen.

Key words: Root canal filling materials. Retreatment. Molar. X-Ray microtomography. 


\section{Introduction}

Endodontic retreatment is indicated when the treatment fails, in the presence of pain, edema, fistula and when the periodontal ligament space is radiographically abnormal ${ }^{1}$. Removing the filling material during retreatment is essential for adequate cleaning, disinfection and shaping of the root canal system (RCS); therefore, this procedure plays an important role in the success of endodontic therapy². During root canal filling, gutta-percha is the main constituent of the obturation material; however, because it does not adhere to dentin walls, it must be used in conjunction with endodontic sealers, which can adhere to canal walls and provide a more homogeneous obturation ${ }^{3}$.

The composition of bioceramic sealers includes calcium silicate, and the sealers are biocompatible, have antimicrobial properties, are radiopaque, chemically stable and do not shrink after setting ${ }^{4-7}$. Due to their chemical composition, they form an interfacial layer with tag-like structures at the cement-dentin interface, which increases the push-out bond strength of those materials ${ }^{8,9}$. However, there is no consensus about the difficulty in removing these sealers after setting ${ }^{10-15}$. Kakoura and Pantelidou ${ }^{13}$ (2018) observed by scanning electron microscopy (SEM) no significant differences in the percentage of the remaining filling material after retreatment of single-rotted teeth filled with gutta-percha and BioRoot, TotalFill BC or AH26. The disadvantages of the methods such as scanning electron microscopy, stereomicroscopy, optical microscopy and digital radiography, that have been used to evaluate the removal of bioceramic sealers from the RCS, include loss of sample, qualitative analysis, and two-dimensional evaluation of images ${ }^{12,16-19}$.

In this context, micro-computed tomography (micro-CT) is a method that has been widely used in recent years to evaluate RCS obturation and retreatment through precise three-dimensional analysis of the root canal and the filling and remaining material

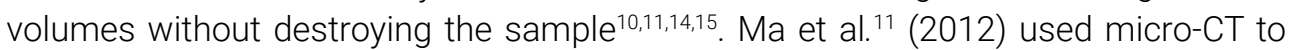
evaluate the removal of the bioceramic iRoot SP with or without solvent from incisors filled by lateral condensation and continuous wave condensation techniques. They found more remaining filling material in the continuous wave condensation group and in the no-solvent groups. Recent studies, also using micro-CT, have shown more remaining filling material in uniradicular teeth obturated with bioceramic sealer, removed with solvent, than that of other sealers ${ }^{10,15}$. According to Agrafioti et al. ${ }^{12}$ (2015), the calcium silicate-based sealers TotalFill BC and MTA Fillapex are negotiable under simple root canal anatomy, but the conventional retreatment techniques are not able to fully remove them. All these studies used single-rooted teeth that present lower anatomical complexity when compared with maxillary and mandibular molars, where clinicians tend to find more difficulty during the retreatment.

Therefore, the objective of the present study was to evaluate, using micro-CT, the removal of the bioceramic TotalFill BC from the mesial roots of mandibular molars using the ProTaper Retreatment instrument without using gutta-percha solvent. The hypothesis is that the bioceramic sealer is harder to remove than the resin-based AH plus. 


\section{Material and Methods}

\section{Specimen selection and initial preparation}

This study was approved by the Research Ethics Committee of of Clementino Fraga Filho Hospital (protocol number 02235718.0.0000.5257). A sample calculation was performed using a test power of 0.8 and an alpha value fixed at 0.05 , which resulted in a minimum of nine samples per group to show statistical significance. Forty mandibular molars with complete root formation, a median length of $19.5 \mathrm{~mm}$ and a root curvature angle of up to $25^{\circ}$, extracted for clinical reasons, were used in this study. The teeth were stored in $0.1 \%$ thymol solution at $4^{\circ} \mathrm{C}$ until used. Access cavities were created with round diamond and Endo-Z burs (Dentsply Maillefer, Ballaigues, Switzerland) in a water-cooled high-speed handpiece. No file was inserted through the mesiobuccal, mesiolingual or distal canals to prevent changes in the original anatomy of the apical region.

\section{Micro-CT scanning}

Micro-CT images were acquired according to the methodology of Almeida et al. ${ }^{20}$ (2015); a custom-made mould of self-polymerizing resin was created for each tooth to standardize the specimen mounting. The teeth were placed in a micro-CT scanner (SkyScan 1173; Bruker microCT, Kontich, Belgium) on a custom aluminium attachment. This attachment ensured high similarity between the images obtained before and after canal preparation because it enabled precise specimen repositioning inside the scanner. Scanning was performed through $360^{\circ}$ rotation with a rotation step of 0.30 using a 1.0-mm thick aluminium filter, $70 \mathrm{kV}$ power, $114 \mathrm{~mA}$ current, $14.8 \mathrm{~mm}$ pixel size and $21.39 \mathrm{~mm}$ resolution.

\section{Root canal instrumentation and obturation}

Coronal third enlargement was performed with LA Axxess stainless steel burs (SybronEndo, Orange, CA, USA), and size $10 \mathrm{~K}$-files (Dentsply Maillefer) were used during instrumentation to establish apical patency and to determine the canal lengths. After radiographic evaluation, the working length was established $1 \mathrm{~mm}$ short of the radiographic apex. The teeth were instrumented to the working length with $\mathrm{K} 3$ files (SybronEndo) using the crown-down technique at $350 \mathrm{rpm}$ with a torque control endodontic motor (Easy, Belo Horizonte, Brazil) using the sequence recommended by the manufacturer as follows: \#25/.08, \#25/.06 and \#25/.04. All files were used passively, and apical enlargement was performed using \#25.06 and \#30.04 files. The pulp chamber was filled with $5.25 \%$ sodium hypochlorite $(\mathrm{NaOCl})$ throughout the instrumentation. The canal was irrigated with $3 \mathrm{ml} 5.25 \% \mathrm{NaOCl}$ after each instrument. After instrumentation, all canals were irrigated with $3 \mathrm{ml}$ 17\% EDTA for 3 minutes ( $1 \mathrm{ml} / \mathrm{min}$ ) and $3 \mathrm{ml} 5.25 \% \mathrm{NaOCl}$, and dried with FM-size paper points (SybronEndo). Next, the teeth underwent postoperative micro-CT scanning with the parameter settings described above. Upon acquisition of the initial images, the specimens were divided into two groups based on their anatomical equivalence according to the number of canal entrances, number of canals, ramifications and isthmus. 
The teeth were divided into two groups of 20 teeth each according to the sealer and technique used: The TotalFill (TF) group used TotalFill BC (FKG Dentaire, La Chauxde-Fonds, Switzerland), \#30 or \#35 gutta-percha cones and TotalFill BC sealer (FKG Dentaire). The AH Plus (AHP) group used size FM or M gutta-percha cones (Dentsply-Maillefer) and AH Plus sealer (Dentsply-Maillefer). In both groups, the cone was placed in the working length. After radiographic certification of the obturation limit, the canals were filled with $5.25 \% \mathrm{NaOCl}$ and underwent passive ultrasonic irrigation (PUI) with a Delsonic 2000 (Deldent, Petah Tikva, Israel) with a \#20 ultrasonic tip and $30-\mathrm{kHz}$ potency for 1 minute. The canals were irrigated with $5 \mathrm{ml}$ distilled water, dried with \#35 paper cones (Dentsply-Maillefer), filled with EDTA for 3 minutes ( $1 \mathrm{ml} / \mathrm{min}$ ), irrigated with $5 \mathrm{ml} 5.25 \% \mathrm{NaOCl}$, washed again with $5 \mathrm{ml}$ distilled water and dried. The obturation technique used was the continuous heat wave. The sealer was introduced into the canal using a \#15 K-file. The gutta-percha cone was covered with sealer and introduced to the working length. System B was used, with the medium tip inserted into the canal $5 \mathrm{~mm}$ short of the working length to remove the gutta-percha from the cervical and middle thirds. The Obtura II system (Obtura, Fenton, MO, USA) was used to fill the middle and cervical thirds with $4-\mathrm{mm}$ increments of gutta-percha at $200^{\circ} \mathrm{C}$.

After filling, the pulp chamber of all teeth was sealed with cotton and zinc oxide-based temporary material, and stored for 7 days in an oven at $37^{\circ} \mathrm{C}$ and $100 \%$ moisture for the sealers' total setting times. Then, the dental elements underwent micro-CT image acquisition again.

\section{Removal of filling material}

The filling material was removed using a \#3 Gates Glidden bur (Dentsply-Maillefer) in the cervical $3 \mathrm{~mm}$ of the canal, followed by the rotary NiTi ProTaper Universal retreatment (PTUR) sequence (Dentsply-Maillefer), as per the manufacturer instructions: D1, D2 and D3 were used in the cervical, middle and apical thirds of the canal until the working length, respectively, without solvent. Filling material removal was considered finished when no gutta-percha or sealer residue was observed in the instrument and when the working length was achieved. After that, a NiTi ProTaper Next X4 (\#40.06, Dentsply-Maillefer) was used until the working length, followed by irrigation with $5 \mathrm{ml}$ $\mathrm{NaOCl} 5.25 \%$. Then, the dental elements underwent micro-CT image acquisition again.

\section{Micro-CT scanning measurements}

Only the mesial roots of the mandibular molars were evaluated. The images were analysed using CTAn software (Bruker microCT). NRecon software (v1.6.1.0, Bruker microCT) was used for the image reconstruction with a $25 \%$ beam hardening correction, ring artifact correction of 2 and smoothing of 2, resulting in the acquisition of 700 to 800 axial sections per sample. The slices of the samples after obturation were recorded with their respective slices after endodontic retreatment by the Affine plugin of the 3D Slicer 4.4.0 program. After the images were recorded, the gray scale range required to recognize the dentin (range 60-255) and the remaining filling material (range 210-255) was determined on a density histogram using a threshold method. Comparisons between acquisitions before and after root canal preparation were made to ensure the accuracy of the segmentation. All image analysis proce- 
dures were performed using the ImageJ 1.50d program (National Institutes of Health, Bethesda, MD, USA). After the reconstruction, the images were analyzed in the CTAn software ( $\mathrm{V}$. 1.16.4.1), where the selection of the region of interest (ROI), binarization and image segmentation for analysis is made. This program provides the necessary morphometric parameters for the proposed study, which was the volume of filling material per third (cervical, middle and apical) and total. The DataViewer (V. 1.5.2.4) and CTvox (V. 3.2.0.0) software were also used for visualization and qualitative analysis of 2D and 3D images of the anatomy of the teeth, respectively.

The images obtained before the chemical-mechanical preparation were used to verify the anatomical equivalence of the groups. The filling volume was determined from the image obtained after obturation, and the total volume of remaining filling material was obtained after retreatment. The difference between these two values was calculated and resulted in $\Delta \mathrm{V}$, which represents the volume of material removed from the RCS. The volumes of filling and remaining material were also determined in the cervical, middle and apical thirds of the canal. The percentage of remaining filling material was calculated based on the formula: Remaining volume $\times 100 \div$ filling volume.

\section{Statistical analysis}

The volume values underwent normality analysis by the Shapiro-Wilk test, and showed normal distribution. The independent $t$-test was used to compare the groups for initial canal volume, volume of obturation, volume of remaining filling and $\Delta \mathrm{V}$. The paired $t$-test was used to compare the volume of obturation and the remaining filling volume within the same group. Analysis of variance (ANOVA) was used to compare the thirds of the canal in terms of the obturation volume and the remaining filling volume within the same group. An alpha value of 0.05 was established as the level of statistical significance (SPSS v20.0, Chicago, IL, USA).

\section{Results}

Table 1 shows the volume of obturation, the volume of remaining filling material, total $\Delta \mathrm{V}$ and $\Delta \mathrm{V}$ in the cervical, middle and apical thirds of the canal. There was no statistical difference between the groups for the volume of obturation, volume of remaining filling material and $\Delta \mathrm{V}$ both in the entire root canal and in the cervical, middle and

Table 1. Mean \pm standard deviation $\left(\mathrm{mm}^{3}\right)$ of obturation volume, remaining filling volume and $\Delta \mathrm{V}$ in the cervical, middle, apical and entire root canal

\begin{tabular}{lcccccc}
\hline & \multicolumn{2}{c}{ AH plus } & \multicolumn{3}{c}{ Total Fill BC sealer } \\
\hline & $\begin{array}{c}\text { Obturation } \\
\text { Volume }\end{array}$ & $\begin{array}{c}\text { Remaining } \\
\text { Filling Volume }\end{array}$ & $\Delta \mathbf{V}$ & $\begin{array}{c}\text { Obturation } \\
\text { Volume }\end{array}$ & $\begin{array}{c}\text { Remaining } \\
\text { Filling Volume }\end{array}$ & $\Delta \mathbf{V}$ \\
\hline Cervical & $5.85 \pm 2.16^{\mathrm{a}}$ & $0.58 \pm 0.46^{\mathrm{e}}$ & $5.28 \pm 2.06^{\mathrm{h}}$ & $6.39 \pm 2.16^{\mathrm{a}}$ & $0.99 \pm 0.85^{\mathrm{e}}$ & $5.39 \pm 2.13^{\mathrm{h}}$ \\
\hline Middle & $3.25 \pm 1.19^{\mathrm{b}}$ & $1.06 \pm 0.77^{\mathrm{f}}$ & $2.18 \pm 0.95^{\mathrm{i}}$ & $3.65 \pm 1.57^{\mathrm{b}}$ & $1.40 \pm 1.45^{\mathrm{e}, \mathrm{f}}$ & $2.25 \pm 1.70^{\mathrm{i}}$ \\
\hline Apical & $1.36 \pm 0.89^{\mathrm{c}}$ & $0.80 \pm 0.78^{\mathrm{e}, \mathrm{f}}$ & $0.55 \pm 0.46^{\mathrm{j}}$ & $1.47 \pm 0.75^{\mathrm{c}}$ & $0.80 \pm 0.88^{\mathrm{e}, \mathrm{f}}$ & $0.66 \pm 0.76^{\mathrm{j}}$ \\
\hline Total & $10.46 \pm 3.79^{\mathrm{d}}$ & $2.44 \pm 1.65^{\mathrm{g}}$ & $8.01 \pm 3.01^{\mathrm{k}}$ & $11.51 \pm 4.01^{\mathrm{d}}$ & $3.19 \pm 2.78^{\mathrm{g}}$ & $8.30 \pm 3.78^{\mathrm{k}}$ \\
\hline
\end{tabular}

* Different letters indicate statistical difference $(p<0.05)$ 
apical thirds of the canal (independent $t$-test, $p>0.05$ ). In both groups, the volume of obturation and volume of remaining filling material were significantly different in the entire root canal and in the three thirds of the canal (paired $t$-test, $p<0.05$ ). However, the filling material was not completely removed in all specimens. In the AHP group, the remaining filling volume in the cervical third was lower than that of the middle third, without significant differences with the apical third (ANOVA, $p<0.05$ ). The TF group showed no significant differences for remaining filling volume in the three thirds of the canal. Patency was not obtained in $35 \%$ and $25 \%$ of the samples in the AHP group and TF group, respectively (ANOVA, $p>0.05$ ).

Figure 1 shows the representative reconstructed micro-CT images of the root canal obturated (Fig. 1a, f), with the remaining filling material (Fig. 1b, g) and transversal sections of the cervical (Fig. 1c, h), middle (Fig. 1d, i) and apical (Fig. 1e, j) thirds of the AHP and TF groups. We noted that the filling material was not completely removed in the groups analysed. In both groups, the presence of remaining filling material was visible in the canal walls and isthmus (Fig. 1c-e, h-j).

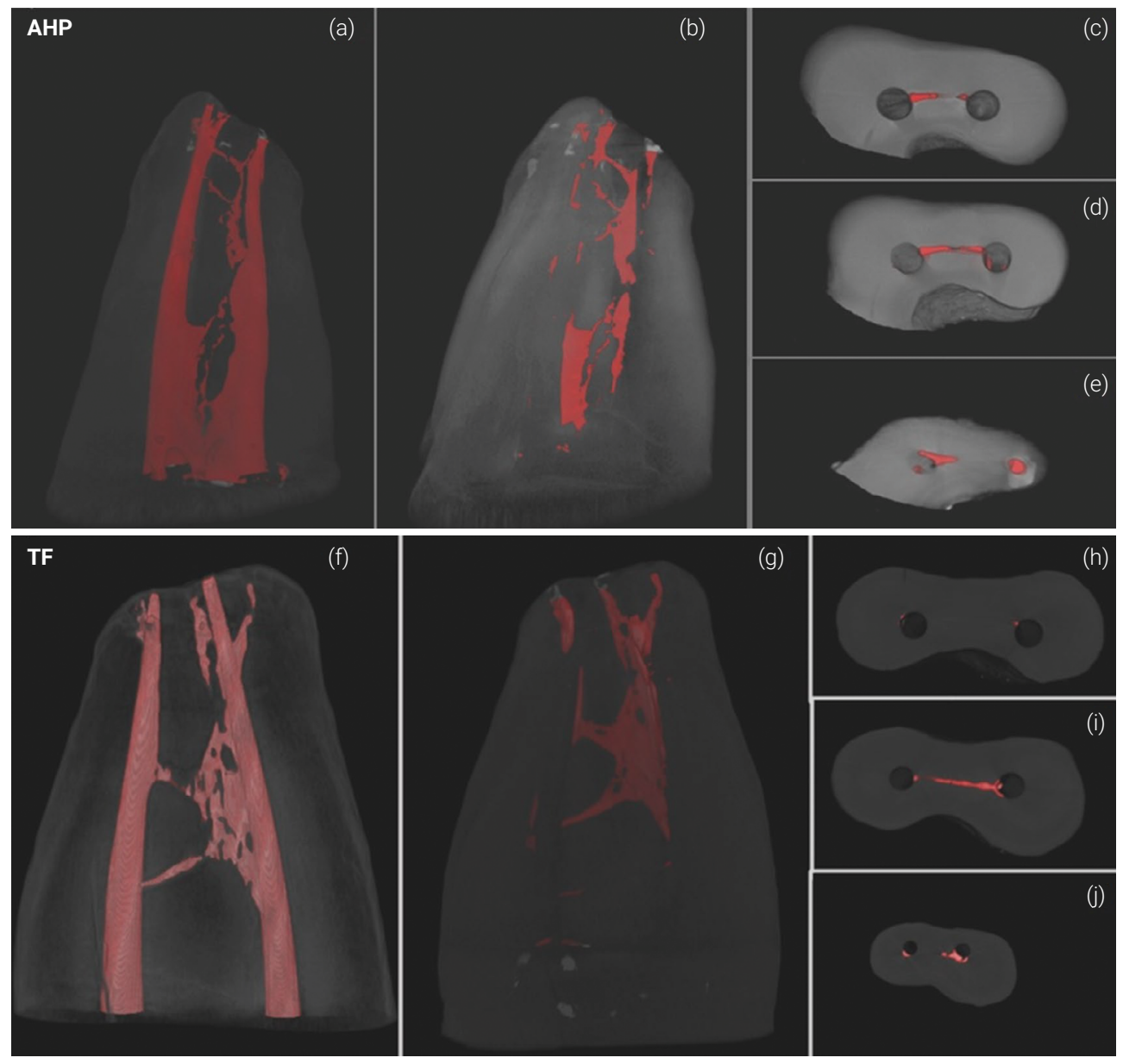

Figure 1. Three-dimensional images of the obturated root canal $(a, f)$, remaining filling material $(b, g)$ and transversal section images of the cervical $(c, h)$, middle $(d, i)$ and apical $(e, j)$ thirds of the AHP and TF groups. 


\section{Discussion}

The present study used micro-CT to evaluate the removal of filling material from the mesial roots of mandibular molars obturated with TotalFill BC or AH Plus sealers using PTUR without gutta-percha solvent. The initial hypothesis has to be rejected since there was no difference between the analysed parameters of both groups. Although the removal of filling material was significant when compared to the volume of obturation, complete removal of filling material from the RCS was not observed in any specimen.

The sealers used in the present study have similar physicochemical characteristics, such as high flow, biocompatibility and radiopacity ${ }^{7,21}$, although their compositions differ and also their adhesion mechanism to dentin. AH Plus is an epoxy resin sealer that interacts chemically with the root dentin collagen with covalent bonds between its epoxy rings and collagen amine groups ${ }^{22}$. Total fill BC presents chemical bonding to mineralized tissues and therefore, the use of EDTA as an irrigating agent to remove smear-layer prior to obturation could decrease the interaction with the dentin wall, since EDTA removes calcium from dentin ${ }^{22,23}$. This could possibly explain the similar results obtained with the sealers used.

Micro-CT is a method that has been widely used in recent years to evaluate RCS anatomy, obturation and retreatment through three-dimensional analysis ${ }^{10,11,15,20,21}$. In the present study, micro-CT showed $23 \%$ remaining filling material in the AHP group, whereas that in the TF group was $28 \%$; the two groups were not statistically significantly different. These results corroborate with other studies that also used micro-CT to evaluate the retreatability of bioceramic sealers, albeit with different methodologies ${ }^{15,17,18}$. Ersev et al. ${ }^{17}$ (2012) used digital radiography to analyse the removal of AH Plus, Hybrid Root SEAL, Activ GP sealers and the EndoSequence BC bioceramic sealer using manual and rotary (ProTaper-R) instruments, while Kim et al. ${ }^{18}$ (2015) used the ProFile rotary instrument to evaluate, by means of MEV, the cleaning of root canals obturated with AH Plus or EndoSequence BC sealers. Oltra et al. ${ }^{15}$ (2017) used micro-CT to evaluate the removal of AH Plus and EndoSequence BC sealers with the ProFile and Vortex Blue rotary instruments, with and without solvent, and observed no difference in the percentage of remaining material in the no-solvent groups. These authors also observed that the bioceramic sealer group had more remaining filling material in the apical third of the canal than the AHP group. Furthermore, patency was not established in $86 \%$ of specimens in the bioceramic group, whereas the AHP group achieved $100 \%$ patency. In the present study, patency was not obtained in 35\% and $25 \%$ of specimens from the AHP and TF groups, respectively. This difference in achieving patency can be related to the anatomic differences of the teeth used in the respective studies. Oltra et al. ${ }^{15}$ (2017) utilized uniradicular teeth with a final apical diameter of \#45.04, whereas we used the mesial canals of mandibular molars with a lower final diameter (\#30.4). The diameter and more circular anatomy of the uniradicular teeth could have favoured more compact filling in the apical third with sealer, making it difficult to remove. On the other hand, in molar teeth, the presence of irregularities and re-entrances in the apical third can lead to empty spaces in the filling mass, which can allow the passage of small-diameter files. In fact, a study that compared root canals filled with bioceramic sealer showed that patency was estab- 
lished in $20 \%$ of the cases obturated in the working length and in only $70 \%$ of cases obturated $2 \mathrm{~mm}$ before the working length ${ }^{16}$.

Our results are not in agreement with other studies that also evaluated the retreatability of bioceramic sealers. Uzunoglu et al. ${ }^{19}$ (2015) used stereomicroscopy and observed more remaining filling material in the group obturated with the bioceramic iRoot SP sealer as compared with the resin-based AH 26 sealer. Agrafioti et al. ${ }^{12}$ (2015) used a clinical microscope to compare the removal of AH Plus, MTA Fillapex and TotalFill BC sealers, and found more remaining filling material in the AH Plus group. Zuolo et al. ${ }^{10}$ (2016) used micro-CT and found more remaining filling material in teeth obturated with EndoSequence BC sealer when compared with a zinc oxide-based sealer. Differences in the methodology and sample used may explain the divergent results.

Rotary instruments are recommended for removing filling material during root canal retreatment because they are fast, safe and efficient. In the present study, PTUR was selected for its proven effectiveness and safety in the extravasation of debris ${ }^{24}$. In fact, our results demonstrate that the volume of remaining filling material was significantly lower than the obturation volume, which agrees with other studies that used the same instrument ${ }^{11,17,19}$. Furthermore, no gutta-percha solvent was used during retreatment, as its use is still controversial in the literature. Although the use of solvent favours the penetration of instruments in the obturation mass, it has been shown by MEV that the dentinal tubules of teeth re-treated with solvents can be obliterated by residues of gutta-percha and sealer, thus making RCS cleaning difficult. Studies that evaluated the influence of the solvent during the retreatment of teeth obturated with bioceramics have shown that filling material removal was either easier or harder when solvent was used ${ }^{11,15}$.

According to the literature, the present study also demonstrates that filling material removal was not complete in any specimen analysed ${ }^{10-12,15,16-19}$. Furthermore, the remaining filling material in the entire root canal and in the three thirds of the canal was higher when compared to other studies that also used micro-CT ${ }^{10,11,15}$. These results may be explained by the anatomical complexity of the mesial canal of the mandibular molars, with high prevalence of isthmus and ramifications, when compared with the uniradicular teeth used in the other studies. Indeed, the AHP group had a higher percentage of remaining filling material in the middle rather than the cervical third of the canal, probably due to the presence of isthmus in that region.

Our findings based on the micro-CT reconstruction images showed the presence of filling material in the re-entrances, isthmus and canal walls, which demonstrates not only how challenging the retreatment of these high anatomical complexity teeth is, but also that the routine procedures during retreatment are ineffective, regardless of the type of filling material used. Therefore, is imperative the need for an additional step of cleaning, as these anatomical areas cannot be reached by the routine procedures that aim at disinfecting the RCS.

\section{References}

1. Consensus report of the European Society of Endodontology on quality guidelines for endodontic treatment. Int Endod J. 1994 May;27(3):115-24. doi: 10.1111/j.1365-2591.1994.tb00240.x. 
2. Olcay K, Ataoglu H, Belli S. Evaluation of related factors in the failure of endodontically treated teeth: A cross-sectional study. J Endod. 2018 Jan;44(1):38-45. doi: 10.1016/j.joen.2017.08.029.

3. Evans JT, Simon JH. Evaluation of the apical seal produced by injected thermoplasticized Guttapercha in the absence of smear layer and root canal sealer. J Endod. 1986;12(3):100-7.

4. Zhang H, Shen $\mathrm{Y}$, Ruse ND, Haapasalo M. Antibacterial activity of endodontic sealers by modified direct contact test against Enterococcus faecalis. J Endod. 2009 Jul;35(7):1051-5. doi: 10.1016/j.joen.2009.04.022.

5. Rodríguez-Lozano FJ, García-Bernal D, Oñate-Sánchez RE, Ortolani-Seltenerich PS, Forner L, Moraleda JM. Evaluation of cytocompatibility of calcium silicate-based endodontic sealers and their effects on the biological responses of mesenchymal dental stem cells. Int Endod J. 2017 Jan;50(1):67-76. doi: 10.1111/iej.12596. Epub 2016 Jan 22.

6. Kapralos V, Koutroulis A, Ørstavik D, Sunde PT, Rukke HV. Antibacterial activity of endodontic sealers against planktonic bacteria and bacteria in biofilms. J Endod. 2018 Jan;44(1):149-54 doi: 10.1016/j.joen.2017.08.023.

7. Tanomaru-Filho M, Torres FFE, Chávez-Andrade GM, de Almeida M, Navarro LG, Steier $\mathrm{L}$, et al. Physicochemical properties and volumetric change of silicone/bioactive glass and calcium silicate-based endodontic sealers. J Endod. 2017 Dec;43(12):2097-101. doi: 10.1016/j.joen.2017.07.005.

8. Viapiana R, Guerreiro-Tanomaru J, Tanomaru-Filho M, Camilleri J. Interface of dentine to root canal sealers. J Dent. 2014 Mar;42(3):336-50. doi: 10.1016/j.jdent.2013.11.013.

9. Reyes-Carmona J F, Felippe M S, Felippe W T. The biomineralization ability of mineral trioxide aggregate and portland cement on dentin enhances the push-out strength. J Endod. 2010 Feb;36(2):286-91. doi: 10.1016/j.joen.2009.10.009.

10. Zuolo A, Zuolo ML, da Silveira Bueno CE, Chu R, Cunha RS. evaluation of the efficacy of TRUShape and Reciproc File Systems in the removal of root filling material: an ex vivo micro-computed tomographic study. J Endod. 2016 Feb;42(2):315-9. doi: 10.1016/j.joen.2015.11.005.

11. Ma J, Al-Ashaw AJ, Shen Y, Gao Y, Yang Y, Zhang C, et al. Efficacy of ProTaper Universal Rotary Retreatment system for gutta-percha removal from oval root canals: a micro-computed tomography study. J Endod. 2012 Nov;38(11):1516-20. doi: 10.1016/j.joen.2012.08.001.

12. Agrafioti $A$, Koursoumis $A D$, Kontakiotis EG. Re-establishing apical patency after obturation with Gutta-percha and two novel calcium silicate-based sealers. Eur J Dent. 2015;9(4):457-61. doi: 10.4103/1305-7456.172625.

13. Kakoura F, Pantelidou O. Retreatability of root canals filled with Gutta percha and a novel bioceramic sealer: a scanning electron microscopy study. J Conserv Dent. 2018;21(6):632-6. doi: 10.4103/JCD.JCD_228_18.

14. Wolf M, Küpper K, Reimann S, Bourauel C, Frentzen M. 3D analyses of interface voids in root canals filled with different sealer materials in combination with warm gutta-percha technique. Clin Oral Investig. 2014 Jan;18(1):155-61. doi: 10.1007/s00784-013-0970-y.

15. Oltra E, Cox TC, LaCourse MR, Johnson JD, Paranjpe A. Retreatability of two endodontic sealers, EndoSequence BC Sealer and AH Plus: a microcomputed tomographic comparison. Restor Dent Endod. 2017 Feb;42(1):19-26. doi: 10.5395/rde.2017.42.1.19.

16. Hess D, Solomon E, Spears R, He J. Retreatability of a bioceramic root canal sealing material. J Endod. 2011 Nov;37(11):1547-9. doi: 10.1016/j.joen.2011.08.016.

17. Ersev H., Yilmaz B., Dinçol M.E., Daglaroglu R. The efficacy of ProTaper universal rotatory retreatment instrumentation to remove single gutta-percha cones cemented with several endodontic sealers. Int Endod J. 2012 Aug;45(8):756-62. doi: 10.1111/j.1365-2591.2012.02032.x. 
18. Kim H., Kim E., Lee SJ, Shin SJ. Comparisons of the retreatment efficacy of calcium silicate and epoxy resin based sealers and residual Sealer in Dentinal Tubules. J Endod. 2015; 41(12): 2025-30.

19. Uzunoglu E, Yilmaz Z, Sungur DD, Altundasar E. Retreatability of root canals obturated using gutta-percha with bioceramic, MTA and resin-based sealers. Iran Endod J. 2015 Dec;41(12):2025-30. doi: 10.1016/j.joen.2015.08.030

20. Almeida BC, Ormiga F, de Araújo MC, Lopes RT, Lima IC, dos Santos BC, et al. Influence of heat treatment of nickel-titanium rotary endodontic instruments on apical preparation: a micro-computed tomographic study. J Endod. 2015 Dec;41(12):2031-5. doi: 10.1016/j.joen.2015.09.001.

21. Roizenblit RN, Soares FO, Lopes RT, Santos BC, Gusman H. Root canal filling quality of mandibular molars with Endosequence BC and AH Plus sealers: a micro-CT study. Aust Endod J. 2020 Apr;46(1):82-7. doi: 10.1111/aej.12373.

22. Crozeta BM, Lopes FC, Menezes Silva R, Silva-Sousa YTC, Moretti LF, Sousa-Neto MD. Retreatability of BC Sealer and AH Plus root canal sealers using new supplementary instrumentation protocol during non-surgical endodontic retreatment. Clin Oral Investig. 2020 Jun 6. doi: 10.1007/s00784-020-03376-4.

23. Soares IMV, Crozeta BM, Pereira RD, Silva RG, da Cruz-Filho AM. Influence of endodontic sealers with different chemical compositions on bond strength of the resin cement/glass fiber post junction to root dentin. Clin Oral Investig. 2020 Oct;24(10):3417-23. doi: 10.1007/s00784-020-03212-9.

24. Çanakçi BC, Ustun Y, Er O, Genc Sen O. Evaluation of apically extruded debris from curved root canal filling removal using 5 Nickel-Titanium Systems. J Endod. 2016 Jul;42(7):1101-4 doi: 10.1016/j.joen.2016.03.012. 